\author{
M.T. Jenaliyev ${ }^{1}$, M.I. Ramazanov ${ }^{2}$, A.A. Assetov² \\ ${ }^{1}$ Institute of Mathematics and Mathematical Modeling, Almaty, Kazakhstan \\ ${ }^{2}$ Ye.A. Buketov Karaganda state university, Kazakhstan \\ (E-mail: muvasharkhan@gmail.com, bekaaskar@mail.ru)
}

\title{
On Solonnikov-Fasano Problem for the Burgers Equation
}

\begin{abstract}
The paper is devoted to the questions of solvability in the Sobolev classes for boundary value problem for the Burgers equation with boundary conditions of the Solonnikov-Fasano type in degenerating domain with degenerate point at the origin. By applying the Galerkin methods and a priori estimates we prove the Existence and Uniqueness Theorems for the solution of the considered boundary value problem, as well as its regularity with increasing smoothness of given functions.
\end{abstract}

Keywords: Burgers equation, boundary value problem, Sobolev classes, degenerating domain, Galerkin methods, a priori estimates.

\section{Introduction}

Studying of the Burgers equation has a long history, part of which is given in the works [1] and [2], and also in the books [3] and [4].

In the works [1] and [2] were studied the solvability of the boundary value problems for the Burgers equation in a non-rectangular domain. If in [1] it is required that it (a non-degenerate domain) be transformed by regularly replacing (independent) variables into a rectangular domain; then in work [2] this requirement is excluded (the domain of independent variables degenerates at the initial moment of time). In Sobolev spaces, by using the Faedo-Galerkin methods and a priori estimates the existence and uniqueness of a regular solution of the boundary value problems under consideration are established.

The paper [5] studies in the angular domain the boundary value problem for the heat equation with the time derivative under boundary conditions. It is also noted there that the case of an nonhomogeneous boundary value problem "... is useful for study of some problems with free boundaries". For example, for single-phase problem "... Stefan under the following assumptions: the liquid phase with a positive temperature $u(x, t)$ occupies the segment $0<x<s(t)$, at $x=0$ a positive heat flow is given, and free boundary $x=s(t)$ starts at the solid boundary $x=0$, i.e. the condition $s(0)=0$ is satisfied". Note that in the paper [5] the theorem on the unique solvability of the considered boundary value problem in weight Holder spaces is established.

The range of application of boundary value problems for parabolic type equations in a domain with a boundary that varies in time is quite wide. Problems of this kind arise: in the study of thermal processes in electrical contacts [6], in the processes of ecology and medicine [7], in the solution of some problems of hydromechanics [8], thermomechanics during heat stroke [9], and so on.

Voluminous literature is devoted to the study of the solvability of linear and nonlinear parabolic equations in cylindrical domains. However, regard to nonlinear boundary value problems in degenerate non-cylindrical domains, they have been studied relatively little.

For angular domains in the Lebesgue classes, we studied boundary value problems of heat conduction and established theorems on their solvability by reducing to the Volterra singular integral equations of the second kind [10], [11].

In [12] we studied various cases of nonhomogeneous boundary. In these cases, it is shown that takes place both unique solvability and non-unique solvability for the corresponding boundary value problems. 
In this paper we study in the Sobolev classes issues of solvability of the boundary value problem for the Burgers equation in angular (degenerate) domain with time derivatives in boundary conditions (in some sense an analogue of the Solonnikov-Fasano problem [5] for the Burgers equation). In Sec. 1, we give the statement of the boundary value problem, with respect to which in Sec. 2 we construct a sequence of boundary value problems in non-degenerate domains. Here, using a transformation of independent variables, we come to a family of problems in the corresponding rectangular domains. A number of theorems on their unique solvability are formulated. Section 3 establishes a priori estimates for solving the above boundary value problems. In the same section, we formulate the main result of the work in the form of a theorem for the initial nonlinear boundary value problems in a degenerate triangular domain. The proofs of these theorems are given in sections 4 and 5 . The work finishes with a brief conclusion.

\section{Statement of the boundary value problem}

Let $Q_{x t_{1}}=\left\{x, t_{1} \mid 0<x<t_{1}, 0<t_{1}<T_{1}<\infty\right\}$ be a triangle domain, one of the vertices of which is at the origin, and $\Omega_{t_{1}}$ be a cross section of the domain $Q_{x t_{1}}$ for fixed temporary variable $t_{1} \in\left(0, T_{1}\right)$. In domain $Q_{x t_{1}}$ we consider the following boundary value problem for the Burgers equation:

$$
\begin{gathered}
\partial_{t_{1}} u+u \partial_{x} u-\nu \partial_{x}^{2} u=f, \nu>0, \\
{\left.\left[\partial_{t_{1}} u-\partial_{x} u\left(x, t_{1}\right)\right]\right|_{x=0}=0,\left.\left[\partial_{t_{1}} u+2 \partial_{x} u\left(x, t_{1}\right)\right]\right|_{x=t_{1}}=0,}
\end{gathered}
$$

where

$$
f \in L_{2}\left(Q_{x t_{1}}\right) \cap C\left(\bar{Q}_{x t_{1}}\right) .
$$

In this paper we study the question of the existence and uniqueness of a solution to a boundary value problem (1)-(2) in the Sobolev space (throughout the paper the notation of spaces corresponds to those adopted in the book [13]):

$$
u \in H^{2,1}\left(Q_{x t_{1}}\right) / X_{x t_{1}} \equiv\left\{L_{2}\left(0, T_{1} ; H^{2}\left(0, t_{1}\right)\right) \cap H^{1}\left(0, T_{1} ; L_{2}\left(0, t_{1}\right)\right)\right\} / X_{x t_{1}},
$$

where (for space $V) V / X_{x t_{1}}$ is a is the quotient space over the subspace $X_{x t_{1}}$ consisting of all possible constants $k=$ const, defined on the set $Q_{x t_{1}}$.

\section{On a family of auxiliary boundary value problems} in quadrangular domains (in the form of trapezoids)

To the problem (1)-(4) we will put a family of boundary value problems, each of which is considered in the domain representing the corresponding trapezoid.

So, let $n \in \mathbb{N}^{*} \equiv\left\{n \in \mathbb{N}: n \geq n_{1}, 1 / n_{1}<T_{1}\right\}, Q_{x t_{1}}^{n}=\left\{x, t_{1}: 0<x<t_{1}, 1 / n<t_{1}<T_{1}<\infty\right\}$ be a trapezoid, and $\Omega_{t_{1}}$ be a cross section of a trapezoid for a given $t_{1} \in\left(1 / n, T_{1}\right)$. Note that at the point $t_{1}=1 / n$ domain $Q_{x t_{1}}^{n}$ no longer degenerates to a point, in addition, between the original domain $Q_{x t_{1}}$ and the domains $Q_{x t_{1}}^{n}$ take place strict inclusions $Q_{x t_{1}}^{n_{1}} \subset Q_{x t_{1}}^{n_{1}+1} \subset \ldots \subset Q_{x t_{1}}$ and, obviously that $\lim _{n \rightarrow \infty} Q_{x t_{1}}^{n}=Q_{x t_{1}}$

In the non-degenerating domain $Q_{x t_{1}}^{n}$ (for each finite $n \in \mathbb{N}^{*}$ ) we consider a boundary value problem:

$$
\begin{gathered}
\partial_{t_{1}} u_{n}+u_{n} \partial_{x} u_{n}-\nu \partial_{x}^{2} u_{n}=f_{n}, \\
{\left.\left[\partial_{t_{1}} u-\partial_{x} u\left(x, t_{1}\right)\right]\right|_{x=0}=0,\left.\left[\partial_{t_{1}} u_{n}+2 \partial_{x} u_{n}\left(x, t_{1}\right)\right]\right|_{x=t_{1}}=0,\left.u_{n}\left(x, t_{1}\right)\right|_{t_{1}=1 / n}=0} \\
f_{n} \in L_{2}\left(Q_{x t_{1}}^{n}\right) \cap C\left(\bar{Q}_{x t_{1}}\right) .
\end{gathered}
$$


We want to transform the boundary value problem (5)-(7) so that it would be placed in a rectangular domain. To do this we will transform the independent variables: let's move from variables $\left\{x, t_{1}\right\}$ to variables $\{y, t\}$. We have that

$$
x=\frac{y}{n-t}, t_{1}=\frac{1}{n-t} ; y=\frac{x}{t_{1}}, t=n-\frac{1}{t_{1}} ;
$$

$Q_{y t}^{n}=\{y, t: 0<y<1,0<t<T\}$ is a rectangular domain, and $\Omega$ is a cross section of the rectangle $Q_{y t}^{n}$ for any fixed $t \in[0, T]$,

$$
t_{1}=1 / n \Leftrightarrow t=0, t_{1}=T_{1} \Leftrightarrow t=T=n-\frac{1}{T_{1}} .
$$

Since

$$
\tilde{u}_{n}(y, t) \triangleq u_{n}\left(\frac{y}{n-t}, \frac{1}{n-t}\right), \tilde{f}_{n}(y, t)=f_{n}\left(\frac{y}{n-t}, \frac{1}{n-t}\right),
$$

then for derivative with respect to $t_{1}$ from function $u_{n}\left(x, t_{1}\right)(9)$ we have that

$$
\partial_{t_{1}} u_{n}\left(x, t_{1}\right)=(n-t)^{2} \partial_{t} \tilde{u}_{n}(y, t)-(n-t) y \partial_{y} \tilde{u}_{n}(y, t) .
$$

Now we find derivatives from function $u_{n}\left(x, t_{1}\right)(9)$ with respect to variable $x$ :

$$
\partial_{x} u_{n}=(n-t) \partial_{y} \tilde{u}_{n}, \quad \partial_{x}^{2} u_{n}=(n-t)^{2} \partial_{y}^{2} \tilde{u}_{n} .
$$

We write down the boundary value problem (5)-(7) in domain $Q_{y t}^{n}$ :

$$
\begin{gathered}
\partial_{t} \tilde{u}_{n}+(n-t)^{-1} \tilde{u}_{n} \partial_{y} \tilde{u}_{n}-\nu \partial_{y}^{2} \tilde{u}_{n}-y(n-t)^{-1} \partial_{y} \tilde{u}_{n}=(n-t)^{-2} \tilde{f}_{n}, \\
{\left.\left[\partial_{t} \tilde{u}_{n}-(n-t)^{-1} \partial_{y} \tilde{u}_{n}(y, t)\right]\right|_{y=0}=0,\left.\quad\left[\partial_{t} \tilde{u}_{n}+(n-t)^{-1} \partial_{y} \tilde{u}_{n}\right]\right|_{y=1}=0,0<t<T,} \\
\tilde{u}_{n}(y, 0)=0, \quad y \in \Omega=\{y: 0<y<1\} .
\end{gathered}
$$

Remark 1. The relations (8)-(9) are one-to-one, i.e. reversible. In the future, we will use this property.

Instead of (10)-(12) in domain $Q_{y t}^{n}$ we will consider more general boundary value problem:

$$
\begin{gathered}
\partial_{t} \tilde{u}_{n}+\alpha_{n}(t) \tilde{u}_{n} \partial_{y} \tilde{u}_{n}-\nu \partial_{y}^{2} \tilde{u}_{n}-\gamma_{n}(y, t) \partial_{y} \tilde{u}_{n}=\beta_{n}(t) \tilde{f}_{n}, \quad(\nu>0), \\
{\left.\left[\partial_{t} \tilde{u}_{n}-\varepsilon_{n}(t) \partial_{y} \tilde{u}_{n}(y, t)\right]\right|_{y=0}=0,\left.\left[\partial_{t} \tilde{u}_{n}+\delta_{n}(t) \partial_{y} \tilde{u}_{n}\right]\right|_{y=1}=0, \quad 0<t<T,} \\
\tilde{u}_{n}(y, 0)=0, \quad y \in \Omega=\{y: 0<y<1\} .
\end{gathered}
$$

where given continuous functions $\alpha_{n}(t), \beta_{n}(t), \gamma_{n}(y, t), \delta_{n}(t)$ for each fixed number $n \in \mathbb{N}^{*}$ satisfy the conditions

$$
\begin{array}{cl}
\alpha_{1 n} \leq \alpha_{n}(t) \leq \alpha_{2 n}, \beta_{1 n} \leq \beta_{n}(t) \leq \beta_{2 n}, \delta_{1 n} \leq\left|\delta_{n}(t)\right| \leq \delta_{2 n}, & \forall t \in[0, T], \\
\varepsilon_{1 n} \leq\left|\varepsilon_{n}(t)\right| \leq \varepsilon_{2 n},\left|\gamma_{n}(y, t)\right| \leq \gamma_{1 n},\left|\partial_{y} \gamma_{n}(y, t)\right| \leq \gamma_{1 n}, & \forall\{y, t\} \in Q_{y t}^{n},
\end{array}
$$

with given positive constants $\alpha_{1 n}, \alpha_{2 n}, \beta_{1 n}, \beta_{2 n}, \delta_{1 n}, \delta_{2 n}, \varepsilon_{1 n}, \varepsilon_{2 n}, \gamma_{1 n}$.

Remark 2. For the coefficients of the boundary value problem (10)-(12) conditions (16) take place and accordingly take the form:

$$
0<\frac{1}{n}=\alpha_{1 n} \leq \alpha_{n}(t)=\frac{1}{n-t} \leq \alpha_{2 n}=T_{1}, 0<\frac{1}{n^{2}}=\beta_{1 n} \leq \beta_{n}(t)=\frac{1}{(n-t)^{2}} \leq \beta_{2 n}=T_{1}^{2},
$$




$$
\begin{gathered}
0<\frac{2}{n}=\delta_{1 n} \leq \delta_{n}(t)=\frac{2}{n-t} \leq \delta_{2 n}=T_{1}, 0<\frac{1}{n}=\varepsilon_{1 n} \leq \varepsilon_{n}(t)=\frac{1}{n-t} \leq \varepsilon_{2 n}=T_{1}, \\
\left|\gamma_{n}(y, t)\right|=\frac{y}{n-t} \leq \gamma_{1 n}=T_{1}, \quad\left|\partial_{y} \gamma_{n}(y, t)\right|=\frac{1}{n-t} \leq \gamma_{1 n}=T_{1} .
\end{gathered}
$$

The following theorem holds.

Theorem 1. Let $n \in \mathbb{N}^{*}$ be a fixed number. Thus, if

$$
\tilde{f}_{n} \in L_{2}\left(Q_{y t}^{n}\right) \cap C\left(\bar{Q}_{y t}^{n}\right)
$$

and

$$
\alpha_{n}(t), \beta_{n}(t), \delta_{n}(t), \gamma_{n}(y, t)
$$

satisfy the conditions (16), then boundary value problem (13)-(15) has a unique solution

$$
\tilde{u}_{n} \in H^{2,1}\left(Q_{y t}^{n}\right) \equiv L_{2}\left(0, T ; H^{2}(0,1)\right) \cap H^{1}\left(0, T ; L_{2}(0,1)\right), \tilde{u}_{n}(1, t), \tilde{u}_{n}(0, t) \in H^{1}(0, T),
$$

which satisfies the estimate:

$$
\left\|\tilde{u}_{n}\right\|_{H^{2,1}\left(Q_{y t}^{n}\right)}+\left\|\tilde{u}_{n}(1, t)\right\|_{H^{1}(0, T)}+\left\|\tilde{u}_{n}(0, t)\right\|_{H^{1}(0, T)} \leq K\left(\left\|\tilde{f}_{n}(y, t)\right\|_{L_{2}\left(Q_{y t}^{n}\right)}, \nu, B_{n}\right),
$$

where $K\left(0, \nu, B_{n}\right)=0, B_{n}=\left\{\alpha_{2 n}, \beta_{2 n}, \gamma_{1 n}, \delta_{2 n}, \varepsilon_{2 n}\right\}$.

From Theorem (1) as a corollary, we obtain the following statement.

Theorem 2. Let $n \in \mathbb{N}^{*}$ be a fixed number. Thus, if $\tilde{f}_{n} \in L_{2}\left(Q_{y t}^{n}\right) \cap C\left(\bar{Q}_{y t}^{n}\right)$, then boundary value problem (10)-(12) has a unique solution

$$
\tilde{u}_{n} \in H^{2,1}\left(Q_{y t}^{n}\right) \equiv L_{2}\left(0, T ; H^{2}(0,1)\right) \cap H^{1}\left(0, T ; L_{2}(0,1)\right), \tilde{u}_{n}(1, t), \tilde{u}_{n}(0, t) \in H^{1}(0, T),
$$

which satisfies the estimate:

$$
\left\|\tilde{u}_{n}\right\|_{H^{2,1}\left(Q_{y t}^{n}\right)}+\left\|\tilde{u}_{n}(1, t)\right\|_{H^{1}(0, T)}+\left\|\tilde{u}_{n}(0, t)\right\|_{H^{1}(0, T)} \leq K\left(\left\|\tilde{f}_{n}(y, t)\right\|_{L_{2}\left(Q_{y t}^{n}\right)}, \nu, B\right),
$$

where $K(0, \nu, B)=0, B=\left\{T_{1}, T_{1}^{2}, T_{1}, T_{1}, T_{1}\right\}$.

On the basis of Lemmas 1, 3 and 4 established in Section 3 below, the proof of the theorem 1 can be carried out by the Galerkin method (for example, like in [13]).

We give the correspondence of function spaces in terms of independent variables $\{y, t\} \in Q_{y t}^{n}$ and $\left\{x, t_{1}\right\} \in Q_{x t_{1}}^{n}$ :

$$
\begin{gathered}
\tilde{f}_{n} \in L_{2}\left(Q_{y t}^{n}\right) \cap C\left(\bar{Q}_{y t}^{n}\right) \Leftrightarrow f_{n} \in L_{2}\left(Q_{x t_{1}}^{n}\right) \cap C\left(\bar{Q}_{x t_{1}}^{n}\right), \\
\tilde{u}_{n}(y, t) \in H^{2,1}\left(Q_{y t}^{n}\right) \Leftrightarrow u_{n}\left(x, t_{1}\right) \in H^{2,1}\left(Q_{x t_{1}}^{n}\right) \equiv L_{2}\left(1 / n, T_{1} ; H^{2}\left(0, t_{1}\right)\right) \cap H^{1}\left(1 / n, T_{1} ; L_{2}\left(0, t_{1}\right)\right) .
\end{gathered}
$$

Further, taking into account the correspondence of spaces (19)-(20), in accordance with Theorem 2, as well as transformation formulas (8)-(9), we can formulate the following statement:

Theorem 3. Let $n \in \mathbb{N}^{*}$ be a fixed number. Thus, if $f_{n} \in L_{2}\left(Q_{x t_{1}}^{n}\right) \cap C\left(\bar{Q}_{x t_{1}}^{n}\right)(19)$, then boundary value problem (5)-(7) has a unique solution $u_{n} \in H^{2,1}\left(Q_{x t_{1}}^{n}\right)(20)$,

$$
u_{n}\left(t_{1}, t_{1}\right), u_{n}\left(0, t_{1}\right) \in H^{1}\left(1 / n, T_{1}\right),
$$

which satisfies the estimate:

$$
\left\|u_{n}\right\|_{H^{2,1}\left(Q_{x t_{1}}^{n}\right)}+\left\|u_{n}\left(t_{1}, t_{1}\right)\right\|_{H^{1}\left(1 / n, T_{1}\right)}+\left\|u_{n}\left(0, t_{1}\right)\right\|_{H^{1}\left(1 / n, T_{1}\right)} \leq K_{0}\left(\left\|f_{n}\left(x, t_{1}\right)\right\|_{L_{2}\left(Q_{x t_{1}}^{n}\right)}, \nu, B\right),
$$

where $K_{0}(0, \nu, B)=0, B=\left\{T_{1}, T_{1}^{2}, T_{1}, T_{1}, T_{1}\right\}$.

Theorem 3 is proved in Section 4. 
3 A priori estimates for solving the problem (13)-(15). Statement of the main result

Here we establish a number of lemmas on the basis of which by using the Galerkin method we can prove the theorems (1)-(2) formulated in the previous section.

Lemma 1. There exists a positive constant $K_{1}$ independent of $\tilde{u}_{n}(y, t)$, such that for all $t \in(0, T]$ the following inequality holds:

$$
\begin{gathered}
\left\|\tilde{u}_{n}(y, t)\right\|_{L_{2}(0,1)}^{2}+\left\|\tilde{u}_{n}(1, t)\right\|_{L_{2}(0,1)}^{2}+\left\|\tilde{u}_{n}(0, t)\right\|_{L_{2}(0,1)}^{2}+ \\
+\int_{0}^{t}\left\|\partial_{y} \tilde{u}_{n}(y, \tau)\right\|_{L_{2}(0,1)}^{2} d \tau \leq K_{1}\left(\left\|\tilde{f}_{n}(y, t)\right\|_{L_{2}\left(Q_{y t}^{n}\right)}, \nu, B_{n}\right),
\end{gathered}
$$

where $K_{1}\left(0, \nu, B_{n}\right)=0, B_{n}=\left\{\alpha_{2 n}, \beta_{2 n}, \gamma_{1 n}, \delta_{2 n}, \varepsilon_{2 n}\right\}$.

Proof. Multiplying equation (13) scalarly in $L_{2}\left(0, t_{1}\right)$ by $\tilde{u}_{n}\left(x, t_{1}\right)$ and taking into account (14), we have that

$$
\begin{gathered}
\frac{1}{2} \frac{d}{d t}\left\|\tilde{u}_{n}(y, t)\right\|_{L_{2}(0,1)}^{2}+\nu\left\|\partial_{y} \tilde{u}_{n}(y, t)\right\|_{L_{2}(0,1)}^{2}= \\
=-\alpha_{n}(t)\left(\tilde{u}_{n}(y, t) \partial_{y} \tilde{u}_{n}(y, t), \tilde{u}_{n}(y, t)\right)+\left(\gamma_{n}(y, t) \partial_{y} \tilde{u}_{n}(y, t), \tilde{u}_{n}(y, t)\right)+ \\
+\beta_{n}(t)\left(\tilde{f}_{n}(y, t), \tilde{u}_{n}(y, t)\right)+\left.\nu \tilde{u}_{n}(y, t) \partial_{y} \tilde{u}_{n}(y, t)\right|_{y=0} ^{y=1}= \\
=-\alpha_{n}(t)\left(\tilde{u}_{n}(y, t) \partial_{y} \tilde{u}_{n}(y, t), \tilde{u}_{n}(y, t)\right)+\left(\gamma_{n}(y, t) \partial_{y} \tilde{u}_{n}(y, t), \tilde{u}_{n}(y, t)\right)+ \\
+\beta_{n}(t)\left(\tilde{f}_{n}(y, t), \tilde{u}_{n}(y, t)\right)-\frac{\nu}{\delta_{n}(t)} \cdot \frac{d}{d t}\left|\tilde{u}_{n}(1, t)\right|^{2}-\frac{\nu}{\varepsilon_{n}(t)} \cdot \frac{d}{d t}\left|\tilde{u}_{n}(0, t)\right|^{2},
\end{gathered}
$$

or

$$
\begin{aligned}
& \frac{1}{2} \frac{d}{d t}\left\|\tilde{u}_{n}(y, t)\right\|_{L_{2}(0,1)}^{2}+\frac{\nu}{\delta_{2 n}} \frac{d}{d t}\left|\tilde{u}_{n}(1, t)\right|^{2}+\frac{\nu}{\varepsilon_{2 n}} \frac{d}{d t}\left|\tilde{u}_{n}(0, t)\right|^{2}+\nu\left\|\partial_{y} \tilde{u}_{n}(y, t)\right\|_{L_{2}(0,1)}^{2} \leq \\
& \leq \alpha_{2 n}\left|\left(\tilde{u}_{n}(y, t) \partial_{y} \tilde{u}_{n}(y, t), \tilde{u}_{n}(y, t)\right)\right|+\gamma_{1 n}\left|\left(\partial_{y} \tilde{u}_{n}(y, t), \tilde{u}_{n}(y, t)\right)\right|+\beta_{2 n}\left|\left(\tilde{f}_{n}(y, t), \tilde{u}_{n}(y, t)\right)\right| .
\end{aligned}
$$

Since

$$
\left(\tilde{u}_{n}(y, t) \partial_{y} \tilde{u}_{n}(y, t), \tilde{u}_{n}(y, t)\right)=\left.\frac{1}{3}\left[\tilde{u}_{n}(y, t)\right]^{3}\right|_{y=0} ^{y=1} \leq \frac{1}{3}\left[\left|\tilde{u}_{n}(1, t)\right|^{3}+\left|\tilde{u}_{n}(0, t)\right|^{3}\right],
$$

then using inequalities (23) and

$$
\begin{aligned}
\gamma_{1 n}\left|\left(\partial_{y} \tilde{u}_{n}(y, t), \tilde{u}_{n}(y, t)\right)\right| & \leq \frac{\nu}{2}\left\|\partial_{y} \tilde{u}_{n}(y, t)\right\|_{L_{2}(0,1)}^{2}+\frac{\gamma_{1 n}^{2}}{2 \nu}\left\|\tilde{u}_{n}(y, t)\right\|_{L_{2}(0,1)}^{2}, \\
\beta_{2 n}\left|\left(\tilde{f}_{n}(y, t), \tilde{u}_{n}(y, t)\right)\right| & \leq \frac{1}{2}\left\|\tilde{f}_{n}(y, t)\right\|_{L_{2}(0,1)}^{2}+\frac{\beta_{2 n}^{2}}{2}\left\|\tilde{u}_{n}(y, t)\right\|_{L_{2}(0,1)}^{2},
\end{aligned}
$$

and integrating the relation (22) from 0 to $t$, taking into account (15) we get that

$$
\tilde{v}_{n}(t)+\int_{0}^{t}\left\|\partial_{y} \tilde{u}_{n}(y, \tau)\right\|_{L_{2}(0,1)}^{2} d \tau \leq A_{3}+A_{2} \int_{0}^{t}\left[\tilde{v}_{n}(\tau)+\left(\tilde{v}_{n}(\tau)\right)^{3 / 2}\right] d \tau .
$$

where

$$
\begin{gathered}
\tilde{v}_{n}(t)=\left\|\tilde{u}_{n}(y, t)\right\|_{L_{2}(0,1)}^{2}+\left|\tilde{u}_{n}(1, t)\right|^{2}+\left|\tilde{u}_{n}(0, t)\right|^{2} \\
A_{1}=\min \left\{1 ; \frac{2 \nu}{\delta_{2 n}} ; \frac{2 \nu}{\varepsilon_{2 n}} ; \nu\right\}, A_{1} A_{2}=\max \left\{\frac{2 \alpha_{2 n}}{3} ; \frac{\gamma_{1 n}^{2}}{\nu}+\beta_{2 n}^{2}\right\},
\end{gathered}
$$




$$
A_{1} A_{3}=\left\|\tilde{f}_{n}(y, \tau)\right\|_{L_{2}\left(Q_{y t}\right)}^{2}
$$

From (24) we will have the following inequalities:

$$
\begin{gathered}
\tilde{v}_{n}(t) \leq A_{3}+A_{2} \int_{0}^{t}\left[\tilde{v}_{n}(\tau)+\left(\tilde{v}_{n}(\tau)\right)^{3 / 2}\right] d \tau, \quad t \in(0, T], \\
\int_{0}^{t}\left\|\partial_{y} \tilde{u}_{n}(y, \tau)\right\|_{L_{2}(0,1)}^{2} d \tau \leq A_{3}+A_{2} \int_{0}^{t}\left[\tilde{v}_{n}(\tau)+\left(\tilde{v}_{n}(\tau)\right)^{3 / 2}\right] d \tau, t \in(0, T] .
\end{gathered}
$$

For inequality (25) we will apply the following Lemma 2 from the work of Bihari I. [14] which we cite in its original formulation.

Lemma 2. Let $Y(t), F(t)$ be a positive continuous functions $a \leq t \leq b$ and $k \geq 0, M \geq 0$ (constants), further $\omega(v)$ be a non-negative non-decreasing continuous function for $v \geq 0$. Then from the inequality

$$
Y(t) \leq V(t) \equiv k+M \int_{a}^{t} F(\tau) \omega(Y(\tau)) d \tau \quad(a \leq \tau \leq b)
$$

it follows the inequality

$$
Y(t) \leq V(t) \leq G^{-1}\left(G(k)+M \int_{a}^{t} F(\tau) d \tau\right) \quad\left(a \leq t \leq b^{\prime}\right),
$$

where $b^{\prime} \leq b$,

$$
G(v)=\int_{v_{0}}^{v} \frac{d w}{\omega(w)}\left(v_{0}>0, v \geq 0\right)
$$

and $v=G^{-1}(\psi)$ is an inverse function for $G(v): v \rightarrow \psi\left(G^{-1}(\psi): \psi \rightarrow v\right.$ exists due to monotonicity $G(v))$.

It is obvious that variable $t$ may belong to a sub-interval $\left(a, b^{\prime}\right)$ from $(a, b)$, so that the argument $\psi=G(k)+M \int_{a}^{t} F(\tau) d \tau$ would belong to the function domain $G^{-1}(\psi)$. Therefore, it may turn out that condition (26) will be satisfied only for $a \leq t \leq b^{\prime}$ with some definable $b^{\prime} \leq b$.

In our case we have

$$
Y(t)=\tilde{v}_{n}(t), k=A_{3}, M=A_{2}, F(t) \equiv 1, \omega(v)=v+v^{3 / 2}, a=0, b=T .
$$

First of all note that by $(27) \omega(v):(0, \infty) \rightarrow(0, \infty)$ is a strictly increasing function. We calculate the integral

$$
\psi=G(v)=\int_{v_{0}}^{v} \frac{d w}{\omega(w)}=\int_{v_{0}}^{v} \frac{d w}{w+w^{3 / 2}}=\left.\ln \left[\frac{w}{(1+2 \sqrt{w})^{2}}\right]\right|_{w=v_{0}} ^{w=v}
$$

Taking into account (28), for the value $\psi$ we have

$$
\psi=\ln \left[\frac{v}{(1+2 \sqrt{v})^{2}}\right]=\ln \left[\frac{A_{3}}{\left(1+2 \sqrt{A_{3}}\right)^{2}}\right]+A_{2} t=G\left(A_{3}\right)+A_{2} t .
$$


Further, to find the inverse function $G^{-1}: \psi \rightarrow v$ it is necessary to solve the following algebraic equation with respect to $v$ :

$$
\frac{v}{(1+2 \sqrt{v})^{2}}=\zeta, \text { where } \zeta=\exp \{\psi\} \geq 0
$$

We reduce the equality (30) to the following quadratic equation $\left(v=z^{2}\right)$

$$
(1-4 \zeta) z^{2}-4 \zeta z-\zeta=0
$$

For the roots $z_{1}$ and $z_{2}$ of equation (31) the inverse functions $G^{-1}: \psi \rightarrow v$ will correspond:

$$
v_{1}=\left\{\begin{array}{ll}
1 / 16, & \text { if } 1-4 \zeta=0, \\
\frac{\zeta}{(1-2 \sqrt{\zeta})^{2}}, & \text { if } 1-4 \zeta \neq 0,
\end{array} \quad v_{2}=\frac{\zeta}{(1+2 \sqrt{\zeta})^{2}} \text { for } \zeta \geq 0,\right.
$$

where $\zeta=\exp \{\psi\} \geq 0$.

The first inverse function from (32) is non-negative on $[0, \infty)$, but is not suitable for our purposes, since it has a discontinuity of the second kind at $1-2 \sqrt{\zeta}=0$. The second is devoid of this feature, it is continuous and bounded everywhere on $[0, \infty)$. Hence,

$$
v=G^{-1}(\psi)=\frac{\exp \{\psi\}}{(1+2 \exp \{\psi / 2\})^{2}} .
$$

Now from (33) and (29) we have that

$$
v=G^{-1}(\psi)=G^{-1}\left(G\left(A_{3}\right)+A_{2} t\right)=G^{-1}\left(\ln \left[\frac{A_{3}}{\left(1+2 \sqrt{A_{3}}\right)^{2}}\right]+A_{2} t\right),
$$

i.e.

$$
v=\frac{A^{2} \exp \left\{A_{2} t\right\}}{4\left(1+A \exp \left\{A_{2} t / 2\right\}\right)}, \quad \text { where } 0 \leq A \equiv \frac{2 \sqrt{A_{3}}}{1+2 \sqrt{A_{3}}}<\infty .
$$

Now, applying the inequality (26) from Lemma 2 for (25), we have the estimate

$$
\begin{gathered}
\tilde{v}_{n}(t) \leq \frac{A^{2} \exp \left\{A_{2} t\right\}}{4\left(1+A \exp \left\{A_{2} t / 2\right\}\right)} \leq \\
\leq \max _{0 \leq t \leq T} \frac{A^{2} \exp \left\{A_{2} t\right\}}{4\left(1+A \exp \left\{A_{2} t / 2\right\}\right)} \equiv C_{1}\left(\left\|f_{\nu}(y, t)\right\|_{L_{2}\left(Q_{y t}\right)}, \nu, B_{n}\right), t \in(0, T] .
\end{gathered}
$$

It remains to get the estimate for the summand $\int_{0}^{t}\left\|\partial_{y} \tilde{u}_{n}(y, \tau)\right\|_{L_{2}(0,1)}^{2} d \tau$. On the basis of estimate (34) we get

$$
\int_{0}^{t}\left\|\partial_{y} \tilde{u}_{n}(y, \tau)\right\|_{L_{2}(0,1)}^{2} d \tau \leq C_{2}\left(\left\|f_{\nu}(y, t)\right\|_{L_{2}\left(Q_{y t}\right)}, \nu, B_{n}\right), \quad t \in(0, T] .
$$

Note that constants $C_{1}$ and $C_{2}$ in estimates (34)-(35) satisfy conditions

$$
C_{1}\left(0, \nu, B_{n}\right)=0, C_{2}\left(0, \nu, B_{n}\right)=0 .
$$

Therefore, estimates (34)-(35) complete the proof of the Lemma 1.

Lemma 3. For the positive constant $K_{2}$ independent of $\tilde{u}_{n}(y, t)$, for all $t \in(0, T]$ the following inequality holds:

$$
\left\|\partial_{y} \tilde{u}_{n}(y, t)\right\|_{L_{2}(0,1)}^{2}+\int_{0}^{t}\left|\partial_{y} \tilde{u}_{n}(1, \tau)\right|^{2} d \tau+\int_{0}^{t}\left|\partial_{y} \tilde{u}_{n}(0, \tau)\right|^{2} d \tau+
$$




$$
+\int_{0}^{t}\left\|\partial_{y}^{2} \tilde{u}_{n}(y, \tau)\right\|_{L_{2}(0,1)}^{2} d \tau \leq K_{2}\left(\left\|\tilde{f}_{n}(y, t)\right\|_{L_{2}\left(Q_{y t}^{n}\right)}, \nu, B_{n}\right),
$$

where $K_{2}\left(0, \nu, B_{n}\right)=0, B_{n}=\left\{\alpha_{2 n}, \beta_{2 n}, \gamma_{1 n}, \delta_{2 n}, \varepsilon_{2 n}\right\}$.

Proof. Multiplying equation (13) scalarly in $L_{2}\left(0, t_{1}\right)$ by $-\partial_{x}^{2} \tilde{u}_{n}\left(x, t_{1}\right)$ and taking into account (14), we have that

$$
\begin{gathered}
\frac{1}{2} \frac{d}{d t}\left\|\partial_{y} \tilde{u}_{n}(y, t)\right\|_{L_{2}(0,1)}^{2}+\nu\left\|\partial_{y}^{2} \tilde{u}_{n}(y, t)\right\|_{L_{2}(0,1)}^{2}= \\
=\alpha_{n}(t)\left(\tilde{u}_{n}(y, t) \partial_{y} \tilde{u}_{n}(y, t), \partial_{y}^{2} \tilde{u}_{n}(y, t)\right)-\left(\gamma_{n}(y, t) \partial_{y} \tilde{u}_{n}(y, t), \partial_{y}^{2} \tilde{u}_{n}(y, t)\right)- \\
-\beta_{n}(t)\left(\tilde{f}_{n}(y, t), \partial_{y}^{2} \tilde{u}_{n}(y, t)\right)+\left.\partial_{t} \tilde{u}_{n}(y, t) \partial_{y} \tilde{u}_{n}(y, t)\right|_{y=0} ^{y=1}= \\
=\alpha_{n}(t)\left(\tilde{u}_{n}(y, t) \partial_{y} \tilde{u}_{n}(y, t), \partial_{y}^{2} \tilde{u}_{n}(y, t)\right)-\left(\gamma_{n}(y, t) \partial_{y} \tilde{u}_{n}(y, t), \partial_{y}^{2} \tilde{u}_{n}(y, t)\right)- \\
-\beta_{n}(t)\left(\tilde{f}_{n}(y, t), \partial_{y}^{2} \tilde{u}_{n}(y, t)\right)-\frac{1}{\delta_{2 n}}\left|\partial_{t} \tilde{u}_{n}(1, t)\right|^{2}-\frac{1}{\varepsilon_{2 n}}\left|\partial_{t} \tilde{u}_{n}(0, t)\right|^{2},
\end{gathered}
$$

or

$$
\begin{aligned}
& \frac{1}{2} \frac{d}{d t}\left\|\partial_{y} \tilde{u}_{n}(y, t)\right\|_{L_{2}(0,1)}^{2}+\frac{1}{\delta_{2 n}}\left|\partial_{t} \tilde{u}_{n}(1, t)\right|^{2}+\frac{1}{\varepsilon_{2 n}}\left|\partial_{t} \tilde{u}_{n}(0, t)\right|^{2}+ \\
& +\nu\left\|\partial_{y}^{2} \tilde{u}_{n}(y, t)\right\|_{L_{2}(0,1)}^{2} \leq \alpha_{2 n}\left|\left(\tilde{u}_{n}(y, t) \partial_{y} \tilde{u}_{n}(y, t), \partial_{y}^{2} \tilde{u}_{n}(y, t)\right)\right|+ \\
& +\gamma_{1 n}\left|\left(\partial_{y} \tilde{u}_{n}(y, t), \partial_{y}^{2} \tilde{u}_{n}(y, t)\right)\right|+\beta_{2 n}\left|\left(\tilde{f}_{n}(y, t), \partial_{y}^{2} \tilde{u}_{n}(y, t)\right)\right| .
\end{aligned}
$$

First we consider estimate of nonlinear summand from (37). First, we have

$$
\left|\left(\tilde{u}_{n}(y, t) \partial_{y} \tilde{u}_{n}(y, t), \partial_{y}^{2} \tilde{u}_{n}(y, t)\right)\right| \leq\left\|\tilde{u}_{n}(y, t)\right\|_{L_{4}(0,1)}\left\|\partial_{y} \tilde{u}_{n}(y, t)\right\|_{H^{1}(0,1)}\left\|\partial_{y} \tilde{u}_{n}(y, t)\right\|_{L_{4}(0,1)} .
$$

Next, given the interpolation inequality from ([15], Theorems 5.8-5.9, p.140-141)

$$
\alpha_{2 n}\left\|\partial_{y} \tilde{u}_{n}(y, t)\right\|_{L_{4}(0,1)} \leq C\left\|\partial_{y} \tilde{u}_{n}(y, t)\right\|_{H^{1}(0,1)}^{1 / 2}\left\|\partial_{y} \tilde{u}_{n}(y, t)\right\|_{L_{2}(0,1)}^{1 / 2}, \forall \partial_{y} \tilde{u}_{n}(y, t) \in H^{1}(0,1),
$$

from (38) we get

$$
\begin{gathered}
\alpha_{2 n}\left|\left(\tilde{u}_{n}(y, t) \partial_{y} \tilde{u}_{n}(y, t), \partial_{y}^{2} \tilde{u}_{n}(y, t)\right)\right| \leq \\
\leq C\left\|\tilde{u}_{n}(y, t)\right\|_{L_{4}(0,1)}\left\|\partial_{y} \tilde{u}_{n}(y, t)\right\|_{H^{1}(0,1)}^{3 / 2}\left\|\partial_{y} \tilde{u}_{n}(y, t)\right\|_{L_{2}(0,1)}^{1 / 2} \leq \\
\leq \frac{\nu}{8}\left\|\partial_{y}^{2} \tilde{u}_{n}(y, t)\right\|_{L_{2}(0,1)}^{2}+\left[\frac{\nu}{8}+C_{2}\left\|\tilde{u}_{n}(y, t)\right\|_{L_{4}(0,1)}^{4}\right]\left\|\partial_{y} \tilde{u}_{n}(y, t)\right\|_{L_{2}(0,1)}^{2} .
\end{gathered}
$$

Here we used Young's inequality $\left(p^{-1}+q^{-1}=1\right)$ :

$$
|A B|=\left|\left(a^{1 / p} A\right)\left(a^{1 / q} \frac{B}{a}\right)\right| \leq \frac{a}{p}|A|^{p}+\frac{a}{q a^{q}}|B|^{q},
$$

where

$$
A=\left\|\partial_{y} \tilde{u}_{n}(y, t)\right\|_{H^{1}(0,1)}^{3 / 2}, \quad B=C\left\|\tilde{u}_{n}(y, t)\right\|_{L_{4}(0,1)}\left\|\partial_{y} \tilde{u}_{n}(y, t)\right\|_{L_{2}(0,1)}^{1 / 2}, \quad a=\frac{\nu}{6}, \quad p=\frac{4}{3}, \quad q=4 .
$$

Next, for the last two summands from (37) we have:

$$
\gamma_{1 n}\left|\left(\partial_{y} \tilde{u}_{n}(y, t), \partial_{y}^{2} \tilde{u}_{n}(y, t)\right)\right| \leq \frac{\nu}{8}\left\|\partial_{y}^{2} \tilde{u}_{n}(y, t)\right\|_{L_{2}(0,1)}^{2}+C_{3}\left\|\partial_{y} \tilde{u}_{n}(y, t)\right\|_{L_{2}(0,1)}^{2},
$$




$$
\beta_{2 n}\left|\left(\tilde{f}_{n}(y, t), \partial_{y}^{2} \tilde{u}_{n}(y, t)\right)\right| \leq \frac{\nu}{4}\left\|\partial_{y}^{2} \tilde{u}_{n}(y, t)\right\|_{L_{2}(0,1)}^{2}+C_{4}\left\|\tilde{f}_{n}(y, t)\right\|_{L_{2}(0,1)}^{2} .
$$

From (37), (39)-(40) we get

$$
\begin{aligned}
& \frac{d}{d t}\left\|\partial_{y} \tilde{u}_{n}(y, t)\right\|_{L_{2}(0,1)}^{2}+\frac{2}{\delta_{2 n}}\left|\partial_{t} \tilde{u}_{n}(1, t)\right|^{2}+\frac{2}{\varepsilon_{2 n}}\left|\partial_{t} \tilde{u}_{n}(0, t)\right|^{2}+\nu\left\|\partial_{y}^{2} \tilde{u}_{n}(y, t)\right\|_{L_{2}(0,1)}^{2} \leq \\
& \quad \leq 2 C_{4}\left\|\tilde{f}_{n}(y, t)\right\|_{L_{2}(0,1)}^{2}+\left[\frac{\nu}{4}+2 C_{2}\left\|\tilde{u}_{n}(y, t)\right\|_{L_{4}(0,1)}^{4}+2 C_{3}\right]\left\|\partial_{y} \tilde{u}_{n}(y, t)\right\|_{L_{2}(0,1)}^{2},
\end{aligned}
$$

or, integrating (41) on $t$ from 0 to $t$, we obtain

$$
\begin{gathered}
\left\|\partial_{y} \tilde{u}_{n}(y, t)\right\|_{L_{2}(0,1)}^{2}+\frac{2}{\delta_{2 n}} \int_{0}^{t}\left|\partial_{\tau} \tilde{u}_{n}(1, \tau)\right|^{2} d \tau+\frac{2}{\varepsilon_{2 n}} \int_{0}^{t}\left|\partial_{\tau} \tilde{u}_{n}(0, \tau)\right|^{2} d \tau+ \\
+\nu \int_{0}^{t}\left\|\partial_{y}^{2} \tilde{u}_{n}(y, \tau)\right\|_{L_{2}(0,1)}^{2} d \tau \leq 2 C_{4}\left\|\tilde{f}_{n}(y, t)\right\|_{L_{2}\left(Q_{y t}^{n}\right)}^{2}+\int_{0}^{t} A_{5}(\tau)\left\|\partial_{y} \tilde{u}_{n}(y, \tau)\right\|_{L_{2}(0,1)}^{2} d \tau,
\end{gathered}
$$

where

$$
A_{4}=2 C_{4}, A_{5}(t)=\frac{\nu}{4}+2 C_{2}\left\|\tilde{u}_{n}(y, t)\right\|_{L_{4}(0,1)}^{4}+2 C_{3} .
$$

From inequality (42) in the same way as in the proof of the Lemma 1 we get the required estimate (36). Lemma 3 is completely proved.

Lemma 4. For the positive constants $\left\{K_{3}, K_{4}, K_{5}\right\}$ independent of $\left\{\tilde{u}_{n}(y, t), \tilde{u}_{n}(1, t), \tilde{u}_{n}(0, t)\right\}$, for all $t \in(0, T]$ the following inequalities hold:

$$
\begin{gathered}
\left\|\partial_{t} \tilde{u}_{n}(y, t)\right\|_{L_{2}\left(Q_{y t}^{n}\right)}^{2} \leq K_{3}\left(\left\|\tilde{f}_{n}(y, t)\right\|_{L_{2}\left(Q_{y t}^{n}\right)}, \nu, B_{n}\right), \\
\left\|\partial_{t} \tilde{u}_{n}(1, t)\right\|_{L_{2}(0,1)}^{2} \leq K_{4}\left(\left\|\tilde{f}_{n}(y, t)\right\|_{L_{2}\left(Q_{y t}^{n}\right)}, \nu, B_{n}\right), \\
\left\|\partial_{t} \tilde{u}_{n}(0, t)\right\|_{L_{2}(0,1)}^{2} \leq K_{5}\left(\left\|\tilde{f}_{n}(y, t)\right\|_{L_{2}\left(Q_{y t}^{n}\right)}, \nu, B_{n}\right),
\end{gathered}
$$

where $K_{3}\left(0, \nu, B_{n}\right)=0, K_{4}\left(0, \nu, B_{n}\right)=0, K_{5}\left(0, \nu, B_{n}\right)=0, B_{n}=\left\{\alpha_{2 n}, \beta_{2 n}, \gamma_{1 n}, \delta_{2 n}, \varepsilon_{2 n}\right\}$.

Proof. The statement of the Lemma 4 follows from Lemma 1 and Lemma 3 , as well as from equation (13) and boundary conditions (14).

Therefore, applying the Galerkin method [13], and using the Lemmas (1), (3) and (4) we directly obtain the validity of the statement of Theorem 1 and a priori estimate (17), and with them the validity of the Theorem 2 and a priori estimate (18).

Now we can formulate the main result of our work.

Theorem 4 (Main result). Let $f\left(x, t_{1}\right) \in L_{2}\left(Q_{x t_{1}}\right) \cap C\left(\bar{Q}_{x t_{1}}\right)$. Then problem (1)-(2) has a unique solution (4)

$$
u\left(x, t_{1}\right) \in H^{2,1}\left(Q_{x t_{1}}\right) / X_{x t_{1}} .
$$

Moreover, traces of the solution satisfy the conditions $u\left(t_{1}, t_{1}\right), u\left(0, t_{1}\right) \in H^{1}\left(0, T_{1}\right)$.

Proof of the Theorem 4 will be given below (section 3 ).

\section{Proof of the Theorem 3}

Here we establish a series of lemmas, on the basis of which we will prove the Theorem 3 formulated in Section 2. The following three lemmas are consequences of the lemmas 1,3-4, respectively. 
Lemma 5. There exists a positive constant $K_{1}$ independent of $u_{n}\left(x, t_{1}\right)$, such that for all $t_{1} \in\left(1 / n, T_{1}\right]$ the following inequality holds:

$$
\begin{aligned}
& \left\|u_{n}\left(x, t_{1}\right)\right\|_{L_{2}\left(0, t_{1}\right)}^{2}+\left\|u_{n}\left(t_{1}, t_{1}\right)\right\|_{L_{2}\left(0, t_{1}\right)}^{2}+\left\|u_{n}\left(0, t_{1}\right)\right\|_{L_{2}\left(0, t_{1}\right)}^{2}+ \\
& +\int_{1 / n}^{t_{1}}\left\|\partial_{x} u_{n}\left(x, \tau_{1}\right)\right\|_{L_{2}\left(0, t_{1}\right)}^{2} d \tau_{1} \leq K_{1}\left(\left\|f_{n}\left(x, t_{1}\right)\right\|_{L_{2}\left(Q_{x t_{1}}^{n}\right)}, \nu, B\right),
\end{aligned}
$$

where $K_{1}(0, \nu, B)=0, B=\left\{T_{1}, T_{1}^{2}, T_{1}, T_{1}, T_{1}\right\}$.

Lemma 6. For a positive constant $K_{2}$ independent of $u_{n}\left(x, t_{1}\right)$, for all $t_{1} \in\left(1 / n, T_{1}\right]$ the following inequality holds:

$$
\begin{gathered}
\left\|\partial_{x} u_{n}\left(x, t_{1}\right)\right\|_{L_{2}\left(0, t_{1}\right)}^{2}+\left|\partial_{x} u_{n}\left(t_{1}, t_{1}\right)\right|^{2}+\left|\partial_{x} u_{n}\left(0, t_{1}\right)\right|^{2}+ \\
+\int_{1 / n}^{t_{1}}\left\|\partial_{x}^{2} u_{n}\left(x, \tau_{1}\right)\right\|_{L_{2}\left(1 / n, t_{1}\right)}^{2} d \tau_{1} \leq K_{2}\left(\left\|f_{n}\left(x, t_{1}\right)\right\|_{L_{2}\left(Q_{x t_{1}}^{n}\right)}, \nu, B\right),
\end{gathered}
$$

where $K_{2}(0, \nu, B)=0, B=\left\{T_{1}, T_{1}^{2}, T_{1}, T_{1}, T_{1}\right\}$.

Lemma \%. For positive constants $\left\{K_{3}, K_{4}, K_{5}\right\}$ independent of $\left\{u_{n}\left(x, t_{1}\right), u_{n}\left(t_{1}, t_{1}\right), u_{n}\left(0, t_{1}\right)\right\}$, for all $t_{1} \in\left(1 / n, T_{1}\right]$ the following inequalities hold:

$$
\begin{gathered}
\left\|\partial_{t_{1}} u_{n}\left(x, t_{1}\right)\right\|_{L_{2}\left(Q_{x t_{1}}^{n}\right)}^{2} \leq K_{3}\left(\left\|f_{n}\left(x, t_{1}\right)\right\|_{L_{2}\left(Q_{x t_{1}}^{n}\right)}, \nu, B\right), \\
\left\|\partial_{t_{1}} u_{n}\left(t_{1}, t_{1}\right)\right\|_{L_{2}\left(0, t_{1}\right)}^{2} \leq K_{4}\left(\left\|f_{n}\left(x, t_{1}\right)\right\|_{L_{2}\left(Q_{x t_{1}}^{n}\right)}, \nu, B\right), \\
\left\|\partial_{t} u_{n}\left(0, t_{1}\right)\right\|_{L_{2}\left(0, t_{1}\right)}^{2} \leq K_{5}\left(\left\|f_{n}\left(x, t_{1}\right)\right\|_{L_{2}\left(Q_{x t_{1}}^{n}\right)}, \nu, B\right),
\end{gathered}
$$

where $K_{3}(0, \nu, B)=0, K_{4}(0, \nu, B)=0, K_{5}(0, \nu, B)=0, B=\left\{T_{1}, T_{1}^{2}, T_{1}, T_{1}, T_{1}\right\}$.

Based on the statements of the Lemmas 5-7, using the Galerkin method [13], we establish the validity of the Theorem 3 .

\section{Proof of the Theorem 4}

The proof of Theorem 4 is based on Theorem 3. In boundary value problems (5)-(7) each element of sequences

$$
\left\{u_{n}\left(x, t_{1}\right), f_{n}\left(x, t_{1}\right),\left\{x, t_{1}\right\} \in Q_{x t_{1}}^{n} ; u_{n}\left(t_{1}, t_{1}\right), u_{n}\left(0, t_{1}\right), t_{1} \in\right.
$$

$\left.\left(1 / n, T_{1}\right) ; n \in \mathbb{N}^{*}\right\}$ continue with zero, respectively, over the entire triangle domain $Q_{x t_{1}}$ and the interval $\left(0, T_{1}\right)$. As a result, we obtain a sequence of functions denoted by

$$
\left\{\widetilde{u_{n}\left(x, t_{1}\right)}, \widetilde{f_{n}\left(x, t_{1}\right)}, \widetilde{u_{n}\left(t_{1}, t_{1}\right)}, \widetilde{u_{n}\left(0, t_{1}\right)}, n \in \mathbb{N}^{*}\right\} .
$$

Obviously, each four functions from the sequence (43) satisfies the boundary value problem (1)-(2) according to the statement of the Theorem 3. In addition, we note that estimate (21) will be strengthened if its on right side $\left\|\widehat{f_{n}\left(x, t_{1}\right)}\right\|_{L_{2}\left(Q_{x t_{1}}\right)}$ is replaced to expression $\left\|f\left(x, t_{1}\right)\right\|_{L_{2}\left(Q_{x t_{1}}\right)}$, since

$$
K_{0}\left(\left\|\widetilde{f_{n}\left(x, t_{1}\right)}\right\|_{L_{2}\left(Q_{x t_{1}}\right)}, \nu, B\right) \leq K_{0}\left(\left\|f\left(x, t_{1}\right)\right\|_{L_{2}\left(Q_{x t_{1}}\right)}, \nu, B\right)
$$

where $K_{0}(0, \nu, B)=0, B=\left\{T_{1}, T_{1}^{2}, T_{1}, T_{1}, T_{1}\right\}$. 
Therefore, we obtain a bounded sequence of functions (43), from which we can be extract a weakly convergent sequence, i.e. (for this subsequence, we keep the notation $n$ for the index). We have that

$$
\begin{aligned}
& \widetilde{u_{n}\left(x, t_{1}\right)} \rightarrow w\left(x, t_{1}\right) \text { weakly in } H^{2,1}\left(Q_{x t_{1}}\right), \\
& \widetilde{u_{n}\left(t_{1}, t_{1}\right)} \rightarrow w\left(t_{1}, t_{1}\right) \text { weakly in } H^{1}\left(0, T_{1}\right), \\
& \widetilde{u_{n}\left(0, t_{1}\right)} \rightarrow w\left(0, t_{1}\right) \text { weakly in } H^{1}\left(0, T_{1}\right) .
\end{aligned}
$$

Since from (44) it follows that

$$
\widetilde{u_{n}\left(x, t_{1}\right)} \rightarrow w\left(x, t_{1}\right) \text { strongly in } L_{2}\left(Q_{x t_{1}}\right),
$$

then by (44)-(47) we can pass to the limit as $n \rightarrow \infty$ in integral identities

$$
\begin{gathered}
0=\int_{Q_{x t_{1}}}\left[\partial_{t_{1}} \widetilde{u_{n}\left(x, t_{1}\right)}+\widetilde{u_{n}\left(x, t_{1}\right)} \partial_{x} \widetilde{u_{n}\left(x, t_{1}\right)}-\nu \partial_{x}^{2} \widetilde{u_{n}\left(x, t_{1}\right)}-\widetilde{f_{n}\left(x, t_{1}\right)}\right] \varphi\left(x, t_{1}\right) d x d t_{1} \rightarrow \\
\int_{Q_{x t_{1}}}\left[\partial_{t_{1}} w\left(x, t_{1}\right)+w\left(x, t_{1}\right) \partial_{x} w\left(x, t_{1}\right)-\nu \partial_{x}^{2} w\left(x, t_{1}\right)-f\left(x, t_{1}\right)\right] \varphi\left(x, t_{1}\right) d x d t_{1}, \quad \forall \varphi \in L_{2}\left(Q_{x t_{1}}\right) \\
\quad 0=\left.\int_{0}^{T_{1}}\left[\partial_{t_{1}} \widetilde{u_{n}\left(x, t_{1}\right)}-\partial_{x} \widetilde{u_{n}\left(x, t_{1}\right)}\right]\right|_{x=0} \varphi_{0}\left(t_{1}\right) d t_{1} \rightarrow \\
\left.\int_{0}^{T_{1}}\left[\partial_{t_{1}} w\left(x, t_{1}\right)-\partial_{x} w\left(x, t_{1}\right)\right]\right|_{x=0} \varphi_{0}\left(t_{1}\right) d t_{1} \forall \varphi_{0} \in L_{2}\left(0, T_{1}\right) \\
\left.\rightarrow \int_{0}^{T_{1}}\left[\partial_{t_{1}} w\left(x, t_{1}\right)-\partial_{x} w\left(x, t_{1}\right)\right]\right|_{x=t_{1}} \varphi_{1}\left(t_{1}\right) d t_{1} \forall \varphi_{1} \in L_{2}\left(0, T_{1}\right)
\end{gathered}
$$

So, we have established that the boundary value problem (1)-(2) has a weak solution $w\left(x, t_{1}\right)$ in the sense of integral identities (48)-(50).

Now we show the uniqueness. Let boundary value problem (1)-(2) has two different solutions $u^{(1)}\left(x, t_{1}\right)$ и $u^{(2)}\left(x, t_{1}\right)$. Then their difference $u\left(x, t_{1}\right)=u^{(1)}\left(x, t_{1}\right)-u^{(2)}\left(x, t_{1}\right)$ will satisfy the homogeneous boundary value problem:

$$
\begin{gathered}
\partial_{t_{1}} u+u \partial_{x} u-\nu \partial_{x}^{2} u=0, \nu>0 \\
{\left.\left[\partial_{t_{1}} u-\partial_{x} u\left(x, t_{1}\right)\right]\right|_{x=0}=0,\left.\left[\partial_{t_{1}} u+2 \partial_{x} u\left(x, t_{1}\right)\right]\right|_{x=t_{1}}=0 .}
\end{gathered}
$$

We establish that the boundary value problem (51)-(52) Hwill not have a non-trivial solution that differs from the constant. It is clear that

$$
u\left(x, t_{1}\right) \in L_{\infty}\left(0, T_{1} ; H^{1}\left(0, t_{1}\right)\right), u\left(t_{1}, t_{1}\right) \text { and } u\left(0, t_{1}\right) \in L_{\infty}\left(0, T_{1}\right)
$$


Multiplying equation (51) by function $u\left(x, t_{1}\right)$ scalarly in $L_{2}\left(0, t_{1}\right)$ and taking into account (52), we obtain

$$
\begin{gathered}
\frac{1}{2} \frac{d}{d t_{1}}\left\|u\left(x, t_{1}\right)\right\|_{L_{2}\left(0, t_{1}\right)}^{2}+\nu \frac{d}{d t_{1}}\left|u\left(t_{1}, t_{1}\right)\right|^{2}+\nu \frac{d}{d t_{1}}\left|u\left(0, t_{1}\right)\right|^{2}+\nu\left\|\partial_{x} u\left(x, t_{1}\right)\right\|_{L_{2}\left(0, t_{1}\right)}^{2}= \\
=-\left(\left[u\left(x, t_{1}\right)\right]^{2}, \partial_{x} u^{(1)}\left(x, t_{1}\right)\right)-\left(u^{(2)}\left(x, t_{1}\right), u\left(x, t_{1}\right) \partial_{x} u\left(x, t_{1}\right)\right) .
\end{gathered}
$$

Integrating by parts

$$
\int_{0}^{t_{1}}\left[u\left(x, t_{1}\right)\right]^{2} \partial_{x} u^{(1)}\left(x, t_{1}\right) d x=\left.\left|u\left(x, t_{1}\right)\right|^{2} u^{(1)}\left(x, t_{1}\right)\right|_{x=0} ^{x=t_{1}}-2 \int_{0}^{t_{1}} u^{(1)}\left(x, t_{1}\right) u\left(x, t_{1}\right) \partial_{x} u\left(x, t_{1}\right) d x,
$$

from (54) we have that

$$
\begin{gathered}
\frac{1}{2} \frac{d}{d t_{1}}\left\|u\left(x, t_{1}\right)\right\|_{L_{2}\left(0, t_{1}\right)}^{2}+\nu \frac{d}{d t_{1}}\left|u\left(t_{1}, t_{1}\right)\right|^{2}+\nu \frac{d}{d t_{1}}\left|u\left(0, t_{1}\right)\right|^{2}+\nu\left\|\partial_{x} u\left(x, t_{1}\right)\right\|_{L_{2}\left(0, t_{1}\right)}^{2}= \\
=u^{(1)}\left(t_{1}, t_{1}\right)\left|u\left(t_{1}, t_{1}\right)\right|^{2}-u^{(1)}\left(0, t_{1}\right)\left|u\left(0, t_{1}\right)\right|^{2}+\int_{0}^{t_{1}}\left[2 u^{(1)}\left(x, t_{1}\right)-u^{(2)}\left(x, t_{1}\right)\right] u\left(x, t_{1}\right) \partial_{x} u\left(x, t_{1}\right) d x .
\end{gathered}
$$

Now we estimate the right side of the relation (55). Using the (53), we obtain

$$
\begin{gathered}
u^{(1)}\left(t_{1}, t_{1}\right)\left|u\left(t_{1}, t_{1}\right)\right|^{2}-u^{(1)}\left(0, t_{1}\right)\left|u\left(0, t_{1}\right)\right|^{2}+\int_{0}^{t_{1}}\left[2 u^{(1)}\left(x, t_{1}\right)-u^{(2)}\left(x, t_{1}\right)\right] u\left(x, t_{1}\right) \partial_{x} u\left(x, t_{1}\right) d x \leq \\
\leq\left\|u^{(1)}\left(t_{1}, t_{1}\right)\right\|_{L \infty\left(0, T_{1}\right)}\left|u\left(t_{1}, t_{1}\right)\right|^{2}+\left\|u^{(1)}\left(0, t_{1}\right)\right\|_{L \infty\left(0, T_{1}\right)}\left|u\left(0, t_{1}\right)\right|^{2}+ \\
+\frac{1}{4 \nu}\left[2\left\|u^{(1)}\left(x, t_{1}\right)\right\|_{L \infty\left(Q_{x t_{1}}\right)}+\left\|u^{(2)}\left(x, t_{1}\right)\right\|_{L \infty\left(Q_{x t_{1}}\right)}\right]^{2}\left\|u\left(x, t_{1}\right)\right\|_{L_{2}\left(0, t_{1}\right)}^{2}+\nu\left\|\partial_{x} u\left(x, t_{1}\right)\right\|_{L_{2}\left(0, t_{1}\right)}^{2} .
\end{gathered}
$$

From here and from (55) we get

$$
v(t) \leq C \int_{0}^{t_{1}} v\left(\tau_{1}\right) d \tau_{1}, \quad \text { т.e. } \quad v(t) \equiv 0, \quad \forall t_{1} \in\left(0, T_{1}\right]
$$

where

$$
\begin{gathered}
v(t)=\min \{1,2 \nu\}\left[\left\|u\left(x, t_{1}\right)\right\|_{L_{2}\left(0, t_{1}\right)}^{2}+\left|u\left(t_{1}, t_{1}\right)\right|^{2}+\left|u\left(0, t_{1}\right)\right|^{2}\right], \\
C=\min \{1,2 \nu\} \max \left\{\frac { 1 } { 2 \nu } \left[2\left\|u^{(1)}\left(x, t_{1}\right)\right\|_{L \infty\left(Q_{x t_{1}}\right)}+\left\|u^{(2)}\left(x, t_{1}\right)\right\|_{\left.L \infty\left(Q_{x t_{1}}\right)\right]^{2},}\right.\right. \\
\left.2\left\|u^{(1)}\left(t_{1}, t_{1}\right)\right\|_{L \infty\left(0, T_{1}\right)}, 2\left\|u^{(1)}\left(0, t_{1}\right)\right\|_{L \infty\left(0, T_{1}\right)}\right\} .
\end{gathered}
$$

Therefore, the uniqueness of the solution of the boundary value problem (1)-(2) is established, and together with it the Theorem 4 is completely proved. 


\section{Conclusion}

In the paper, we established in Sobolev classes the solvability theorems for boundary value problem for the Burgers equation in a degenerating domain with degenerate point at the origin.

The results of the work can be generalized to case when we have a domain of independent variables $Q_{x t_{1}}=\left\{x, t_{1}: 0<x<\varphi\left(t_{1}\right), 0<t_{1}<T_{1}<\infty\right\}$, presented in a curved triangle whose moving side can change according to the rule $x=\varphi\left(t_{1}\right), t_{1} \in\left[0, T_{1}\right]$, and the condition $\varphi(0)=0$ is satisfied. In addition, from the function $\varphi\left(t_{1}\right)$ some natural conditions are required. For example, function $\varphi\left(t_{1}\right)$ must satisfy the following two conditions: $1^{0}$. in a sufficiently small time interval $\left(0, t_{1}^{*}\right)$ function $\varphi\left(t_{1}\right)$ would have a representation $\varphi\left(t_{1}\right)=\mu t_{1}$, where $\mu$ is a given positive constant (in our work it was equal to unity); $2^{0}$. on the interval $\left[t_{1}^{*}, T_{1}\right]$ function $\varphi\left(t_{1}\right)$ would be continuous differentiable and would have the property of monotonicity, providing a one-to-one transformation from independent variables $\left\{x, t_{1}\right\}$ to variables $\{y, t\}$.

\section{Funding}

Supported by the grant projects AP05130928 (2018-2020) and AP05132262 (2018-2020) from the Ministry of Science and Education of the Republic of Kazakhstan.

\section{References}

1 Benia Y. Existence of solutions to Burgers equations in domains that can be transformed into rectangles / Y. Benia, B.K. Sadallah // Electronic Journal of Differential Equations. - 2016. 157. - P. 1-13.

2 Benia Y. Existence of solutions to Burgers equations in a non-parabolic domain / Y. Benia, B.K. Sadallah // Electronic Journal of Differential Equations. - 2018. - 20. - P. 1-13.

3 Burgers J.M. The nonlinear diffusion equation. Asymptotic solutions and statistical problems / J.M. Burgers // Advances in Difference Equations. - Boston, USA: D. Reidel Publishing Company, Dordrecht-Holland. - 1974. - 174 p.

4 Вишик М.И. Математические задачи статистической гидромеханики / М.И. Вишик, А.В. Фурсиков. - М.: Наука, 1980. - 440 с.

5 Солонников В.А. Об одномерной параболической задаче, возникающей при изучении некоторых задач со свободными границами / В.А. Солонников, А. Фазано // Записки научного семинара ПОМИ. - 2000. - 269. - С. 322-338.

6 Ким Е.И. Математические модели тепловых процессов в электрических контактах / Е.И. Ким, В.Т. Омельченко, С.Н. Харин. - Алма-Ата: Наука, 1977. - 236 с.

7 Митропольский Ю.А. Задачи со свободными границами для нелинейного эволюционного уравнения в проблемах металлургии, медицины, экологии / Ю.А. Митропольский, А.А. Березовский, Т.А. Плотницкий // Укр. мат. журн. - 1992. - 44. - № 1. - С. 67-75.

8 Веригин Н.Н. Об одном классе гидромеханических задач для областей с подвижными границами / Н.Н. Веригин // Динамика жидкости со свободными границами. - CO АН СССР: - 1980. - 46. - C. 23-32.

9 Карташов Э.М. Проблема теплового удара в области с движущейся границей на основе новых интегральных соотношений / Э.М. Карташов // Изв. РАН. Энергетика. - 1997. - 4. - C. $122-137$.

10 Amangaliyeva M.M. About Dirichlet boundary value problem for the heat equation in the infinite angular domain / M.M. Amangaliyeva, M.T. Jenaliyev, M.T. Kosmakova, M.I. Ramazanov // Boundary Value Problems. - 2014. - 213. - 21 p. 
11 Amangaliyeva M.M. On one homogeneous problem for the heat equation in an infinite angular domain / M.M. Amangaliyeva, M.T. Jenaliyev, M.T. Kosmakova, M.I. Ramazanov // Sib. Math. Jour. - 2015. - Vol. 56.- 6. - P. 982-995.

12 Amangaliyeva, M.M. On the solvability of nonhomogeneous boundary value problem for the Burgers equation in the angular domain and related integral equations. / M.M. Amangaliyeva, M.T. Jenaliyev, M.T. Kosmakova, M.I. Ramazanov // Springer Proceedings in Mathematics and Statistics. - 2017. - 216. - P. 123-141.

13 Lions J.L. Problemes aux limites non homogenes et applications. / J.L. Lions, E. Magenes // Dunod, Paris. - 1968. - vol. 1. - 374 p.

14 Bihari I. A generalization of a lemma of Bellman and its application to uniqueness problems of differential equations / I. Bihari // Acta Math. Acad. Scient. Hung. VII. - 1956. - 1. - P. 81-94.

15 Adams R. A. Sobolev spaces (2-nd Edition) / R. A. Adams, J. J. F. Fournier. - Elsevier, Amsterdam. - 2003. - vol. 140. - 320 p.

\title{
М.Т. Дженалиев, М.И. Рамазанов, А.А. Асетов
}

\section{Бюргерс теңдеуі үшін Солонников-Фазано есебі туралы}

\begin{abstract}
Мақала нүктеге жойылатын облыста, Солонников-Фазано типті шекаралық шарттармен берілген Бюргерс теңдеуі үшін шекаралық есептің соболевтік кластарында шешілуі сұрақтарына арналған. Облыстың жойылу нүктесі координаталар басында орналасқан. Галеркин және априорлық бағалаулар әдістерін қолдану арқылы қарастырылып отырған шекаралық есептің шешімінің бар болуы және жалғыздығы туралы теоремалар, сонымен қатар берілген функциялардың тегістігі артқанда есептің регулярлығы дәлелденген.
\end{abstract}

Kiлm сөздер: Бюргерс теңдеуі, шекаралық есеп, соболевтік кластар, жойылатын облыс, Галеркин әдісі, априорлық бағалаулар.

\section{М.Т. Дженалиев, М.И. Рамазанов, А.А. Асетов}

\section{О задаче Солонникова-Фазано для уравнения Бюргерса}

Работа посвящена вопросам разрешимости в соболевских классах граничной задачи для уравнения Бюргерса с граничными условиями типа Солонникова-Фазано в вырождающейся области. Точка вырождения области находится в начале координат. Использованием методов Галеркина и априорных оценок доказаны теоремы о существовании и единственности решения рассматриваемой граничной задачи, а также его регулярность при повышении гладкости заданных функций.

Ключевые слова: уравнение Бюргерса, граничная задача, соболевские классы, вырождающаяся область, метод Галеркина, априорные оценки.

\section{References}

1 Benia, Y. \& Sadallah, B.-K. (2016). Existence of solutions to Burgers equations in domains that can be transformed into rectangles. Electronic Journal of Differential Equations, 157, 1-13.

2 Benia, Y. \& Sadallah, B.-K. (2018). Existence of solutions to Burgers equations in a non-parabolic domain. Electronic Journal of Differential Equations, 20, 1-13.

3 Burgers, J.M. (1974). The nonlinear diffusion equation. Asymptotic solutions and statistical problems. Advances in Difference Equations. Boston, USA: D. Reidel Publishing Company, DordrechtHolland, 174. 
4 Vishik, M.I. \& Fursikov, A.V. (1980). Matematicheskie zadachi statisticheskoi hidrodinamiki [Mathematical problems of statistical hydromechanics]. Moscow: Nauka [in Russian].

5 Solonnikov, V.A. \& Fasano, A. (2000). Ob odnomernoi parabolicheskoi zadache, voznikaiushchei pri izuchenii nekotorykh zadach so svobodnymi hranitsami [One-dimensional parabolic problem arising in the study of some free boundary problems]. Zapiski nauchnho seminara POMI, 269 (2000), 322-338 [in Russian].

6 Kim, E.I., Omelchenko, V.T. \& Kharin, S.N. (1977). Matematicheskie modeli teplovykh protsessov $v$ elektricheskikh kontaktakh [Mathematical models of thermal processes in electrical contacts]. Alma-Ata: Nauka [in Russian].

7 Mitropolsky, Yu.A., Berezovsky, A.A. \& Plotnitskiy, T.A. (1992). Zadachi so svobodnymi hranitsami dlia nelineinoho evoliutsionnoho uravneniia v problemakh metallurhii, meditsiny, ekolohii [Problems with free boundaries for the nonlinear evolution equation in metallurgy, medicine, and ecology]. Ukrainskii matematicheskii zhurnal, 44, No. 1, 67-75 [in Russian].

8 Verigin, N.N. (1980). Ob odnom klasse hidromekhanicheskikh zadach dlia oblastei s podvizhnymi hranitsami [On one class of hydromechanical problems for domains with moving boundaries]. Dinamika zhidkosti so svobodnymi hranitsami, 46, 23-32 [in Russian].

9 Kartashov, E.M. (1997). Problema teplovoho udara v oblasti s dvizhushcheisia hranitsei na osnove novykh intehralnykh sootnoshenii [The problem of heat stroke in a domain with a moving boundary based on new integral relations]. Izvestiia RAS. Energetics, 4, 122-137 [in Russian].

10 Amangaliyeva, M.M., Jenaliyev, M.T., Kosmakova, M.T. \& Ramazanov, M.I. (2014). About Dirichlet boundary value problem for the heat equation in the infinite angular domain. Boundary Value Problems, 213, 21 p.

11 Amangaliyeva, M.M., Dzhenaliev, M.T., Kosmakova, M.T. \& Ramazanov, M.I. (2015). On one homogeneous problem for the heat equation in an infinite angular domain Sib. Math. Jour. Vol. 56, 6, 982-995.

12 Amangaliyeva, M.M., Jenaliyev, M.T., Kosmakova, M.T. \& Ramazanov, M.I. (2017). On the solvability of nonhomogeneous boundary value problem for the Burgers equation in the angular domain and related integral equations. Springer Proceedings in Mathematics and Statistics, 216, $123-141$.

13 Lions J.-L. \& Magenes E. (1968). Problemes aux limites non homogenes et applications, Vol. 1. Dunod, Paris, 374.

14 Bihari, I. (1956). A generalization of a lemma of Bellman and its application to uniqueness problems of differential equations. Acta Math. Acad. Scient. Hung. VII, 1, 81-94.

15 Adams, R.A. \& Fournier, J.J.F. (2003). Sobolev spaces (2-nd Ed.), Vol. 140. Elsevier, Amsterdam, 320. 